\title{
RADIOPHARMACEUTICAL TRACERS FOR NEURAL PROGENITOR CELLS
}

Thomas J. Mangner, Ph.D.

Wayne State University, Children's Hospital of Michigan

FG02-03ER63607

\section{PROGRESS REPORT}

For funding period 08/14/2004 - 08/14/2005

The overall objective of this proposal is to synthesize and test radiopharmaceutical tracers as in vivo markers of neural progenitor cells for positron emission tomography (PET) imaging. The following is a description of our progress during the second year of funding 08/15/2004 to 08/15/2005.

Our intial microPET imaging results indicated that none of our intial target tracers showed appreciable brain uptake in normal or seizure-induced (kainic acid-treated) animals. These tracers tested were: 3'-deoxy-3'- $\left[{ }^{18} \mathrm{~F}\right]$ fluorothymidine $\quad\left(\left[{ }^{18} \mathrm{~F}\right] \mathrm{FLT}\right), \quad 1-(2$ '-deoxy-2'-fluoro- $\beta$-D-arabinofuranosyl)-5bromouracil ([$\left.\left.{ }^{18} \mathrm{~F}\right] \mathrm{FBAU}\right)$, and $\mathrm{N}-\left[{ }^{18} \mathrm{~F}\right]$ fluoroacetyl-D-glucosamine (FLAG).

During the second year of funding (08/14/2004 - 08/14/2005), we synthesized and examined more lipophilic "prodrugs" of the ${ }^{18} \mathrm{~F}$-labeled thymidine analogs as a potential way of increasing the delivery of the tracers across the blood-brain-barrier where they could be hydrolyzed to the parent compounds and interact with the ongoing DNA synthesis associated with neurogenesis. We have succeeded in preparing several compounds: 5'-benzoyl-[ $\left.{ }^{18} \mathrm{~F}\right] \mathrm{FLT}$ (FLT-BE) and 3',5'-dibenzoyl-[ $\left.{ }^{18} \mathrm{~F}\right] \mathrm{FBAU}$ (FBAUDB) and have compared their brain uptake to that of their parent compounds. We have also prepared a lipophilic "prodrug" of FLAG, tetraacetyl-N-[ $\left.{ }^{18} \mathrm{~F}\right]$ fluoroacetyl-D-glucosamine (TAFLAG) and have examined its brain uptake in a number of animal models using microPET animal scanning.

As a corollary approach to searching for a marker of neurogenesis, we have also examined the potential uptake of L-[1-11 C]leucine in young rats, normal rats and seizure-induced rats to explore the possible imaging of protein synthesis during neurogenesis.

Technical difficulties associated with the also planned development of the anti-nestin antibody/antitransferrin antibody conjugate have prevented us from making any real progress toward developing a potential PET imaging tracer for neurogenesis based on the concept of tranferrin-assisted transport across the blood-brain-barrier.

As noted in our previous progress report, the brain uptake of our original tracer candidates in normal rats (FLT, n=4; FLT-BE, n=1; FBAU, n=5; FBAU-DB, n=3; FLAG, n=4; LEU, n=5) appeared to be lower than the values necessary to abstract any meaningful data from the scans, in all cases appearing as a "cold spot" in the microPET images. These same results were seen when the tracers were administered to neonatal rats ( $<7$ days old, FLT, $n=2 ;$ FBAU, $n=2 ;$ FBAU-DB, $n=1 ; L E U, n=8)$ and to rats pretreated (6 - 7 days) with kainic acid to induce seizure activity (FLT, $n=2$; FLT-BE, $n=1$; FBAU, $n=2$; FBAUDB, $n=2 ;$ FLAG, $n=4$; TAFLAG, $n=2 ;$ LEU, $n=2$ ).

Although some promise was seen with FBAU-DB in potentially increasing the availability of the parent compound $\left[{ }^{18} \mathrm{~F}\right] \mathrm{FBAU}$ to the brain in animals implanted with brain tumors, there was no improvement in brain accumulation of activity when this compound was administered to either neonatal rats $(n=1)$ or seizure-induced rats $(\mathrm{n}=2)$. The prodrug to FLT, 5'-benzoyl- $\left[{ }^{18} \mathrm{~F}\right] \mathrm{FLT}$, demonstrated no improvement in accumulation of brain activity. Similarly, both FLAG and the prodrug to FLAG, TAFLAG, showed very little brain uptake in normal (FLAG, $n=4$; TAFLAG, $n=1)$ and seizure-induced rats (FLAG, $n=4$; TAFLAG, n=2), with no apparent differences between the two tracers or the animal groups. 
In conclusion, none of the PET tracers we synthesized and examined in seizure-induced rats for potential use in detection of neurogenesis by PET imaging showed any promise in that respect. The primary problem common to all the compounds tested was very low brain uptake. An additional complicating factor could be also related to the relatively small target that neurogenesis would represent; only a very limited amount of neurogenesis would be expected to occur at any one time.

Figure 1. (A) MRI, (B) $\left[{ }^{18} \mathrm{~F}\right]$ FLAG microPET image, and (C) brain region time activity curves from the brain of a rat that had undergone kainic acid administration to induce hippocampal neurogenesis. Arrows denote the location of the hippocampus. Faint tracer uptake seen at location of the hippocampus (B) of the kainic acid treated animals was not seen in controls. The time activitv curve shows annroximatelv 2-fold hioher accumulation in hinnocamnus at 20 min nost-iniection comnared to the Figure 2. MicroPET scans of (A) $\left[{ }^{18} \mathrm{~F}\right] \mathrm{FBAU}$ and $(\mathrm{B})\left[{ }^{18} \mathrm{~F}\right] \mathrm{FBAU}-\mathrm{DB}$, and $(\mathrm{C})$ time activity curves for the two tracers in tumor and brain. FBAU-DB shows higher brain penetration and uptake into proliferating tissue (tumor). 